\title{
Tipos de obsolescencia y formas de combatirla desde el derecho privado*
}

\author{
Types of obsolescence and ways of combating it \\ in private law
}

Mónica García Goldar**

Artículo de investigación

Fecha de recepción: 21 de mayo de 2021

Fecha de aceptación: 21 de julio de 2021

\section{Para citar este artículo:}

García Goldar, M. (2021). Tipos de obsolescencia y formas de combatirla desde el derecho privado. Revista Análisis Jurídico-Político, 3(6), 231-252. https://doi.org/10.22490/26655489.4743

\section{REsumen}

En este trabajo analizaremos las cinco formas de obsolescencia - programada, funcional, psicológica, informática e indirectacomo causas detonantes de la "sociedad de consumo" en que nos hallamos inmersos. También se relacionarán algunas propuestas que pueden tomarse desde el derecho privado y que, a nuestro parecer, contribuirían a luchar contra la obsolescencia y a lograr

\footnotetext{
* Este artículo de investigación se basó parcialmente en un trabajo previo galardonado con el II Premio Universidad Loyola a la Investigación en Desarrollo (2021), se enmarca en la ejecución del proyecto "Nuevos desafíos del derecho de consumo en la era digital", financiado por el programa de ayudas a la etapa postdoctoral de la Xunta de Galicia (Consellería de Educación, Universidade e Formación Profesional).

** Doctora en Derecho por la Universidad de Santiago de Compostela (España). Sus intereses investigativos se han centrado en el derecho de familia, derecho de sucesiones y derecho de consumo. Correo electrónico: monica.garcia@usc.es; Orcid: 0000-0002-3235-292X
} 
modalidades de consumo y producción más sostenibles. Entre esas medidas se incluye la mejora de los deberes de información precontractual, la extensión de los plazos de garantía, el reconocimiento del derecho a la reparación ("right to repair", al que a menudo se alude en inglés como "R2R"), así como la obligación impuesta a comerciantes de actualizar los contenidos o servicios digitales.

Palabras clave: actualizaciones, garantías, información, obsolescencia, reparación.

\section{Abstract}

In this paper we will analyse the five forms of obsolescence - programmed, functional, psychological, technological and indirect- as probable causes of the "consumer society" in which we are immersed. We will also propose some measures that can be taken from private law and which, in our opinion, would contribute to fight against obsolescence and to achieving more sustainable consumption and production methods. These measures include the improvement of pre-contractual information duties, the extension of guarantee periods, the recognition of the right to repair (R2R), as well as the obligation imposed on traders to update digital content or services.

Keywords: guarantees; information; obsolescence; repair; updates.

\section{INTRODUCCIÓN}

Entre los 17 objetivos propuestos en la Agenda 2030 para el Desarrollo Sostenible se encuentra el de garantizar modalidades de consumo y producción sostenibles (ODS 12). Tal propósito no solo es indispensable, sino también urgente, en vista de la contundencia con que algunas instituciones vienen alertando del ritmo acelerado de consumo, de la cultura del descarte y de la alteración del medio ambiente (Martínez y Porcelli, 2016, p. 349). El papa Francisco, en su encíclica Laudato si (2015), afirma que es imposible “[...] sostener el actual nivel de consumo de los países más desarrollados". Y la Unión Europea, en su último Plan de Acción para la Economía Circular (2020), denuncia que “[...] solo tenemos una Tierra, pero en 2050 el consumo mundial será el equivalente al de tres planetas". 
Es difícil determinar el momento exacto en que surge esta "sociedad de consumo", proceso que Bauman (2006) describe como el paso de la sociedad de productores a la sociedad de consumidores. Además, esta transformación no se redujo únicamente a la mayor cantidad de productos o servicios consumidos, sino también a la peor calidad de lo que consumimos: hasta principios del siglo XX, los fabricantes solían producir objetos que la gente necesitaba y cuya cualidad inherente era la durabilidad (consumo); muy al contrario, hoy se producen necesidades que solo se satisfacen con algunos productos mucho menos duraderos (consumismo).

Esta nueva jerarquía de valores deprecia la durabilidad y exalta la fugacidad, la rapidez, el exceso y el desperdicio. Si ello solo tuviese repercusiones positivas en el crecimiento económico, no habría razones para alarmarse. El problema es que el consumismo exacerbado degrada el medio ambiente de —al menos- dos formas distintas: la primera, a través de la extracción masiva de ciertos recursos naturales, como pueden ser, en el caso de la industria tecnológica, los metales e incluso algunos elementos tóxicos (Van Acker, 2019, p. 21); la segunda, porque se multiplican exponencialmente los residuos. Dice Bauman que uno

[...] de los atributos necesarios del objeto de consumo es una especie de codicilo añadido a su certificado de nacimiento en el que se puede leer (en letra pequeña, pero clara y tranquilizadoramente legible): destino final: cubo de la basura. Los desperdicios son los productos finales de toda acción del consumidor. La actual percepción del orden de las cosas es justamente la inversa de la que se tenía en la ya pasada sociedad de productores. Entonces era lo útil — extraído de la materia prima adecuadamente reprocesadalo que se suponía que tenía que ser sólido y duradero, mientras que los residuos y los desechos sobrantes estaban destinados a la eliminación inmediata y al olvido. Hoy, le ha llegado el turno a lo útil de ser fugaz, volátil y efímero (de dejar despejado el terreno para la siguiente generación de productos útiles). Solo los residuos tienden (por desgracia) a ser sólidos y perdurables. Solidez es hoy sinónimo de desperdicio. (2006, p. 120)

En vista de la gravedad que reviste la situación, cabría preguntarse cuáles son las causas detonantes de esta transformación que nos ha 
arrastrado a una sociedad hiperconsumista. En nuestra opinión, no cabe duda de que parte del cambio se debe a la introducción deliberada de ciertas prácticas comerciales deshonestas, como la obsolescencia de los productos, y que nos incitan a acudir al mercado con cada vez mayor frecuencia. A esta y a los diferentes tipos de obsolescencia que existen dedicaremos este estudio, proponiendo soluciones que, a nuestro entender, podrían corregir esta tendencia al sobreconsumo.

La metodología empleada en este trabajo es la propia de la rama de las ciencias jurídicas, basada en el análisis de los textos legales y de la doctrina especializada en la materia. En cuanto a la estructura, el trabajo se desarrolla en tres partes: la primera se dedica a detallar los diferentes tipos de obsolescencia; la segunda, a relacionar algunas propuestas que cabría tomar desde el derecho privado; y la tercera, a formular algunas valoraciones finales.

\section{LOS DIFERENTES TIPOS DE OBSOLESCENCIA}

El término "obsolescencia" comúnmente suele asociarse con el conocido fenómeno de la "obsolescencia programada", que es un término bastante autodescriptivo: se trata de la pérdida de funcionalidad de un producto (obsolescencia) que se introduce en el diseño de manera intencional (programada), para que la vida útil se reduzca considerablemente; se trata, pues, de una caducidad deliberada. El momento de su aparición se remonta a comienzos del siglo XX, cuando un agente inmobiliario propuso su introducción para terminar con la crisis de la Gran Depresión, y en 1954 alcanzó su mayor popularidad cuando Brooks Stevens dio una conferencia sobre la nueva producción en masa (Martínez y Porcelli, 2016, p. 342). Su finalidad es eminentemente económica, pues consiste en

\footnotetext{
[...] asegurar que los consumidores acudan al mercado una y otra vez a adquirir productos semejantes más actuales y renovados que presten la misma funcionalidad -o una similar-, al observar que aquellos que ya poseen han devenido obsoletos. Las empresas logran así, mediante este estímulo singular, incrementar la tasa de reposición de los bienes que producen y aumentar los beneficios de todas las industrias. (Soto Pineda, 2015, p. 3)
} 
Este tipo de obsolescencia tan ampliamente extendida debería preocuparnos profundamente, ya no solo porque constituye un riesgo para el medio ambiente, sino también un aminoramiento de los derechos de los consumidores; resulta loable, por ello, que en Francia se haya tipificado como delito (Santamaría Arinas, 2019, p. 3).

Pero si bien esta forma de obsolescencia es la más manifiesta para la sociedad en general, desde luego no es la única; existen otras formas, algunas mucho menos conocidas, pero igual de perniciosas. En The Waste makers (1960, David MackKay Publishers), el sociólogo Vance Packard distinguió, además de la referida obsolescencia de calidad o planificada, la funcional y la de deseabilidad.

La "obsolescencia técnica o funcional" es la que se da cuando un producto queda desfasado porque se introduce otro en el mercado que funciona mejor. Aunque sí es cierto que también contribuye a generar residuos, no parece que sea esta la forma de obsolescencia que más deba preocupar. Según Vidalenc y Meunier (2014, pp. 14-17), cabría distinguir dos tipos de productos: en primer lugar, los que generan un $80 \%$ del impacto ambiental en la etapa de su fabricación - como pueden ser los ordenadores, los smartphones o la vestimenta- $\mathrm{y}$, en segundo lugar, los que generan un $80 \%$ del impacto ambiental en la etapa de su utilización - por ejemplo, los electrodomésticos-. En opinión de los autores referidos, si una nueva tecnología permite economizar el gasto energético anual, sería interesante acelerar el recambio y no alargar la vida de ese producto; es decir, que para este último tipo de productos - electrodomésticos-, la obsolescencia funcional no debe ser valorada necesariamente como un fenómeno negativo.

De otra parte, la "obsolescencia estética, psicológica o de deseabilidad", que se produce en nuestras mentes cuando, a pesar de que el producto sigue funcionando correctamente, pasamos a considerarlo anticuado. Constituye uno de los tipos de obsolescencia más complicados por cuanto es más difícil - por no decir imposible- de sancionar. De hecho, existen estudios que demuestran la importancia de la obsolescencia psicológica y de la cultura social de usar y tirar como barrera a la reparación (Richter y Dalhammar, 2019, p. 5).

Parece que Packard supo ver que tal vez era esta forma de obsolescencia la que más debía preocupar, pues a ella le dedicó un capítulo entero de su obra. Para este autor no cabe duda de que la sensación 
de desapego que se genera en algunos consumidores proviene de los propios comerciantes, que a menudo incorporan pequeños cambios de estilo para que aquellos sientan la necesidad de tener la más reciente versión. La obsolescencia psicológica se interrelaciona de manera acusada con el fenómeno social de las modas: en la moda, como en la modernidad, lo nuevo es preferido a lo viejo.

Una de las funciones de la moda es precisamente atribuir a los objetos un valor simbólico que, adecuado a las referencias culturales de la sociedad, permite al consumidor la afirmación de su personalidad (Mesacasa et ál., 2010, pp. 2-4). Por ello la obsolescencia psicológica es común en la industria de la moda, pero las principales empresas de tecnología, imitando a aquella, llevan años produciendo modelos nuevos con algún añadido que aparentemente los hace más novedosos (Martínez y Porcelli, 2016, p. 347; Soto Pineda, 2015, p. 3). ¿Y quién decide cuál es la moda en uno u otro momento? Pues principalmente los comerciantes y sus campañas de marketing, dirigidas a crear en el consumidor un imperioso deseo por productos que aparejan la misma funcionalidad en un "envoltorio más novedoso" (Soto Pineda, 2015, p. 3).

Lo cierto es que las estrategias de marketing han ido evolucionando con el propio devenir de los años y cada vez se vuelven más y más sofisticadas. Tómese de ejemplo la publicidad a través de las redes sociales y los denominados "influencers", que sirven de escaparate para llegar a un público (target) mucho más específico. Algo similar afirma Nascimento Pereira (2017, p. 213), al considerar que en la actualidad también se venden estilos de vida. Y qué decir de las cookies y de la propaganda que aparece en nuestros dispositivos electrónicos según nuestros historiales de búsquedas en la red. En esta tesitura, los consumidores no deben ser vistos como agentes responsables, sino como colectivo social que debe ser tutelado por el ordenamiento jurídico. Como dicen Pantzar y Suljada (2020, p. 13), es difícil hacer responsables a los consumidores del cambio transformacional mientras las palancas del mercado y de la sociedad sigan incentivándoles a incrementar sus niveles de consumo.

Además de estas tres formas de obsolescencia — programada, psicológica y funcional - de las que hablaba Packard, actualmente se han distinguido dos nuevos tipos más: la obsolescencia informática y la obsolescencia indirecta (Comité Económico y Social Europeo, 2013). 
La "obsolescencia por incompatibilidad o informática" es la que se produce cuando, por ejemplo, un programa informático deja de funcionar al actualizarse el sistema operativo. Por su parte, la "obsolescencia indirecta" es la que deriva de la imposibilidad de reparar un producto por falta de piezas de recambio adecuadas o por resultar imposible la reparación. No cabe duda de que este último tipo de obsolescencia también repercute negativamente en el sobreconsumismo. Un claro ejemplo lo encontramos en la multinacional Apple, cuya política empresarial se ha visto cuestionada desde 2001 por ser contraria a la protección de consumidores, la defensa de la competencia y la protección medioambiental. Soto Pineda $(2015$, p. 4) trae a colación algunas de sus conductas más problemáticas, como las modificaciones que la multinacional hizo de los tornillos de algunos de sus productos o los adaptadores de corriente, que son modificados de forma reiterada; también menciona el asunto Westley vs. Apple Computer Inc. (año 2003), relativo a la duración limitada de las baterías de litio de los iPod de las dos primeras generaciones y la imposibilidad de refacción de estos.

Estas son, a nuestro entender, las cinco formas de obsolescencia que los legisladores de los países más desarrollados deben tener en cuenta a la hora de emprender reformas de calado en materia de sostenibilidad. Según Cooper (2004, p. 442), cabría distinguir también lo que él denomina la "obsolescencia económica", que es la que tiene lugar cuando los propietarios atribuyen poco o ningún valor a un aparato existente y creen que ya no vale la pena mantenerlo en uso, influenciados por el coste de un nuevo modelo de sustitución - por ser más eficiente energéticamente o más económico de mantener- o por el coste de las reparaciones. No obstante, en nuestra opinión, esta obsolescencia económica no es sino una combinación entre la obsolescencia funcional - existe un aparato en el mercado que funciona mejor-y la indirecta — es imposible o excesivamente oneroso reparar el aparato-.

\section{SOluciones DESDE EL DERECHO PRIVAdO}

Una vez referidas las formas de obsolescencia, pasamos a analizar algunas medidas que cabría adoptar y que podrían ser, en algunos casos, versátiles, por cuanto podrían contribuir a reparar los 
perjuicios que se derivan a veces no solo de un tipo, sino de varios tipos de obsolescencia. Dichas propuestas se enumeran siguiendo un criterio temporal: en primer lugar, la puesta del producto a la venta -importancia de la información precontractual-; en segundo lugar, las "deseables" obligaciones posventa —durabilidad para productos físicos; actualizaciones para contenidos o servicios digitales-.

\subsection{Deberes de información SObre duRABilidad/Reparabilidad}

A escala europea, son dos las directivas que se pueden resaltar porque establecen ciertos deberes de información: de una parte, la Directiva 2005/29/CE prohíbe las omisiones engañosas de información sustancial (artículo 7); de otra parte, la Directiva 2011 / 83/UE establece ciertos deberes de información precontractual en sus artículos 5 y 6 . Pero en ninguno de los dos instrumentos legales se establece la obligación del vendedor de informar acerca de la durabilidad/reparabilidad de los productos, ni siquiera tras la reciente modificación llevada a cabo por la Directiva 2019/2161 (Mak y Terryn, 2020, p. 239). Esta ausencia de reforma es especialmente criticable si se tiene en cuenta que la armonización, en algunos casos - como ocurre con los deberes de información precontractual en los contratos a distancia o los celebrados fuera de establecimiento- es total y no permite que los Estados miembros adopten o mantengan requisitos adicionales de información contractual.

Por fortuna, no todos los deberes de información precontractual se han armonizado totalmente - como ocurre en los casos de venta presencial-, lo que ha permitido que en Francia se apruebe la "Loi n” 2020-105 relative à la lutte contre le gaspillage et à l'économie circulaire". Uno de los cuatro grandes pilares sobre los que gira esta ley de 130 artículos es, precisamente, la mejora de la información facilitada ex lege a los consumidores; información que irá resultando exigible en diferentes tiempos. Así, por ejemplo, desde el 1 de enero de 2021, los vendedores de equipos eléctricos y electrónicos deberán mostrar un índice de reparabilidad sobre sus productos, por lo que el consumidor será capaz de saber si el producto es reparable o no.

A partir del 1 de enero de 2022, también se impondrá la puesta a disposición del público de información relativa a los productos que 
contienen perturbadores endocrinos; los operadores de internet y telefonía deberán informar a los clientes sobre el coste de carbono de su consumo digital; y también se informará al consumidor de la garantía legal de conformidad. Dicha información deberá ser mencionada en la factura del producto o el ticket de caja. A partir del 1 de enero de 2024, algunos productos eléctricos o electrónicos -que serán determinados por decreto- deberán mostrar un índice de durabilidad. Resulta claro, en nuestra opinión, que el legislador francés ha asumido con esta ley una indudable posición de liderazgo en esta materia.

Volviendo al nivel comunitario, cabría tener en cuenta que el acceso a cierto tipo de información constituye una demanda real. Como indica Mazeika (2019, p. 12), los resultados preliminares de un estudio realizado por la Unión Europea muestran el deseo de los consumidores por recibir mejor información sobre la durabilidad/reparabilidad de los productos, al ser este uno de los factores más decisivos - junto con el precio y la calidad - a la hora de comprar (Consumer Market Study to support the Fitness Check of EU Consumer and marketing law, 2017, pp. 50-51). La importancia de este factor deriva del hecho de que la durabilidad de un producto les podría hacer ahorrar dinero a largo plazo; es decir, para los consumidores no se trata de una cuestión de economía circular, sino de economía doméstica. Además, y como señalan Terryn y Van Gool (2020, pp. 4-5), si bien es posible que la obligación de información precontractual podría no afectar más que a una minoría de consumidores comprometidos con el medio ambiente, sí que podría contribuir a concienciar a los consumidores, poco a poco, sobre el impacto medioambiental de sus decisiones.

En nuestra opinión, la imposición de deberes claros de información sobre la durabilidad y la reparabilidad de los productos resultaría muy útil en la lucha contra varios tipos de obsolescencia, principalmente la psicológica. Una vez que dicha información sobre durabilidad / reparabilidad resulte disponible a todos los consumidores, serán estos quienes elijan comprar uno u otro producto. No habiendo gran diferencia en el precio, es posible predecir una mayor tendencia a la compra de productos duraderos, pues, al fin y al cabo, y como dijimos, se trata de una cuestión de economía doméstica. Además, un deber de información sobre la durabilidad y la reparabilidad también podría tener un efecto indirecto en los comerciantes que, previendo la competencia 
con otros y la posible preferencia de los consumidores por productos rentables, terminen mejorando la calidad de sus productos.

Por ello, resulta esperanzador que en el nuevo Plan de Acción para la Economía Circular de 2020 se prevea una próxima revisión de la legislación de protección de los consumidores que garantice que estos reciban "[...] información fiable y pertinente sobre los productos que incluya datos sobre su vida útil y sobre la disponibilidad de servicios de reparación, piezas de recambio y manuales de reparación". Además, la Resolución del Parlamento Europeo, "Hacia un mercado único más sostenible para las empresas y los consumidores (2020)", en una sección titulada "Lucha contra la obsolescencia programada y derechos del consumidor", pide a la comisión que elabore una estrategia amplia para, entre otras cuestiones, adoptar estas dos medidas: 1) especificar la información precontractual que debe facilitarse, de manera clara y comprensible, sobre la vida útil estimada y la reparabilidad de un producto - información que deberá incluirse como una de las características principales de un producto en el sentido de las Directivas 2005/29/CE y 2011/83/UE—; y 2) desarrollar e introducir un etiquetado obligatorio donde se informe al consumidor sobre la vida útil estimada y la reparabilidad de un producto. Tal vez se deba precisamente a esta resolución que el Ministerio de Consumo de España haya anunciado, con ocasión del Día del Consumidor del presente año — 15 de marzo de 2021 - que se está trabajando en un índice de reparabilidad para productos eléctricos y electrónicos.

\subsection{Deberes para productos físicos (I): la durabilidad}

Para luchar eficazmente contra la obsolescencia de los productos se debe partir de un término clave, el de durabilidad. Este concepto debe estar al frente de todas las propuestas legislativas venideras y que no solo se circunscriban al derecho privado, sino a todas las ramas jurídicas, incluidas, cómo no, las relativas al ecodesign de los productos. Es decir, los productos ya deben diseñarse y fabricarse con unos criterios de calidad que los hagan mucho más duraderos. Pero además de esas normas que afecten la producción y el diseño, también son importantes las normas que impongan esa producción sostenible de una manera indirecta, a través de las garantías legales. 
A escala europea, las garantías legales se regulan en la reciente Directiva 2019/771, relativa a determinados aspectos de los contratos de compraventa de bienes, y que ha introducido la durabilidad como un nuevo criterio objetivo de conformidad. No obstante, la definición que se hace de ese concepto como "capacidad de los bienes de mantener sus funciones y rendimiento requeridos en condiciones normales de utilización" no resulta del todo satisfactoria, por cuanto no se hace mención al paso del tiempo, como cabría esperar en atención al Behavioural Study on consumers' Engagement in the Circular Economy (Comisión Europea, 2018, pp. 110-114): los consumidores asocian mayoritariamente la durabilidad con los productos que duran y se pueden usar en perfectas condiciones durante bastante tiempo.

Cabría tener en cuenta, además, las importantes observaciones expuestas por Sánchez García (2017) respecto de la interrelación entre la obsolescencia programada y los criterios objetivos de conformidad. Tal interrelación se basa en la exigencia por parte de la normativa europea de que los bienes de consumo presenten la calidad y la durabilidad 1) que presentan normalmente bienes del mismo tipo y 2) que el consumidor pueda razonablemente esperar

[...] dada la naturaleza de los bienes y teniendo en cuenta cualquier declaración pública realizada por el vendedor, o en su nombre, por otras personas en fases previas de la cadena de transacciones, incluido el productor, especialmente en la publicidad o el etiquetado. (pp. 173-193).

Pues bien, el "principio de calidad media" que se deriva de la exigencia de que los bienes posean las cualidades y otras características "que presenten bienes del mismo tipo" podría estar determinado por el tiempo medio de obsolescencia programada con el que fue diseñado. Sánchez García (2017) lo explica así:

Imaginemos, por ejemplo, una reclamación o demanda en torno a la poca vida útil de una pila o una bombilla. Es claro que en estos casos los productos deberán funcionar el tiempo medio que generalmente esté programado para su obsolescencia, no pudiendo tomar como referencia una bombilla con una duración mucho más larga 
o excesivamente corta. [...] Es decir, que conceptos como vida útil media o la naturaleza del producto, como determinantes de la responsabilidad por parte del vendedor en cuanto a la obsolescencia, estarían relacionados y directamente ligados al conocimiento general y usual de la obsolescencia específica del mismo. (pp. 173-174)

En páginas posteriores, el autor vuelve a incidir en este asunto al afirmar:

[...] es paradójico cómo este principio [de conformidad objetiva] no se amolda al fenómeno de la obsolescencia. En efecto, si la calidad del producto se determinara por las cualidades de otros productos similares o iguales en el mercado, y que como dijimos es un fenómeno generalizado que se manifiesta en todos los productos de la misma especie - todos los iPod tenían problemas de las baterías de litio-, se llegaría a la conclusión de que el bien con problemas de obsolescencia estaría conforme al contrato celebrado [...]. (Sánchez García, 2017, p. 188)

Además, y respecto de que los bienes deban presentar las cualidades y características "[...] teniendo en cuenta cualquier declaración pública realizada por el vendedor, o en su nombre, por otras personas en fases previas de la cadena de transacciones, incluido el productor, especialmente en la publicidad o el etiquetado", Sánchez García (2017, pp. 189-193) considera que el vendedor — productor o proveedor- no está obligado, por lo menos de manera específica, a manifestar la obsolescencia y mucho menos a que las empresas presenten en su publicidad los defectos que se puedan generar por ella, por lo que cabría pensar "[...] que querer un bien sin obsolescencia es un requerimiento o uso especial del mismo. Es decir, un uso al que ordinariamente no se destinan los bienes de la clase adquirida por los consumidores". De ello se deriva que la falta de conformidad no desempeñaría ningún papel esencial en dos casos: "[...] por un lado, cuando al momento de celebración del contrato el comprador tenga conocimiento de la obsolescencia; y, por el otro, cuando no pueda de manera fundada ignorarla teniendo en cuenta otros productos similares o información previa".

Por lo tanto, el concepto de conformidad objetiva no representa, en su configuración actual, un mecanismo realmente eficaz, ya que 
"hace conformes" todos los productos con obsolescencia programada, debido a lo extendida que está esta práctica — el consumidor actual intuye que los productos caducarán prematuramente-. Además, la introducción del nuevo criterio de durabilidad no altera en modo alguno los plazos de garantía, por lo que tampoco ha de considerarse que suponga ningún avance en la materia (García Goldar, 2021, pp. 99-100).

Más importante parece el tema de los plazos de garantía legal. En este sentido, cabe lamentar que la Unión Europea haya mantenido un enfoque conservador en la reciente Directiva 2019/771 al no ampliar los plazos, que siguen siendo de dos años (artículo 10). Aunque, por fortuna, la versión final de la directiva sí permite que los Estados miembros amplíen los plazos - al contrario de lo establecido en la propuesta inicial de 2015; cuestión que fue muy criticada por la doctrina, al considerar que se trataba de una disposición poco coherente con los objetivos de sostenibilidad y que no incentivaba a los productores a producir bienes que durasen más de dos años-(Loos, 2017, p. 21).

Sería aconsejable, entonces, que los legisladores nacionales decidiesen hacer uso de esta opción para extender los plazos de garantía: cuantos más Estados miembros así lo establezcan, más incentivos tendrán los productores para producir bienes que efectivamente sean más duraderos. Pero dicha extensión se puede hacer de muchas formas, como por ejemplo: ampliar los plazos de forma general; ampliar los plazos en función de los bienes; establecer nuevos períodos de garantía tras la reparación o la sustitución; ampliar el plazo solo respecto de la reparación; o, incluso, establecer una presunción iuris tantum de que si el producto deja de funcionar tras la expiración del plazo de garantía, es porque se ha diseñado con obsolescencia programada (García Goldar, 2021, pp. 104-109).

Las dos primeras propuestas referidas se analizan en el estudio elaborado en nombre del Consumer Policy Evaluation Consortium, titulado Study on the costs and benefits of extending certain rights under the Consumer Sales and Guarantees Directive 1999/94/EC (Comisión Europea, Dirección General de Justicia y Consumidores, 2017, pp. 61-62). En cuanto a la primera, se dice que un plazo de garantía de tres o de cinco años sería más beneficioso para los consumidores y con él se abordaría el problema de la obsolescencia programada, 
aunque una medida de ese calibre supondría costes más elevados para las empresas. Sobre esto último, Pazos (2020, p. 214) afirma que, cuánto más duradero sea un bien y más caro sea el precio que deba pagarse, menos incentivos parece que tendrán los consumidores para elegir los bienes sostenibles. Sin embargo, un informe del Eurobarómetro sobre Attitudes of Europeans towards building the single market for green products (Comisión Europea, 2012, p. 108) revela que el $66 \%$ de los consumidores europeos estarían dispuestos a pagar más si la garantía de un producto se extendiese hasta los cinco años, y según el Consumer Market Study to support the Fitness Check of EU Consumer and marketing law (2017, pp. 50-51), los consumidores estarían dispuestos a pagar más por productos con una mayor durabilidad.

En cuanto a la segunda propuesta, relativa a establecer a nivel europeo un período de garantía vinculado a la vida útil promedio de los bienes - la solución preferible en opinión de cierta doctrina- (Keirsbilck et ál., 2020, p. 20), cabe tener en cuenta que según el citado Study on the costs and benefits of extending certain rights under the Consumer Sales and Guarantees Directive 1999/44/EC (Comisión Europea, Dirección General de Justicia y Consumidores, 2017, pp. 61-62), el sistema de los Países Bajos se considera opaco, complejo y una carga en términos de quejas y remedios. En Finlandia, sin embargo, la opinión parece ser menos negativa, tal vez porque es un sistema que está en vigor desde 1978 y al que todas las partes implicadas parecen estar acostumbradas. A pesar de la posible judicialización a la que podría llevar esta medida - al menos en el momento actual, en el que no existen estándares uniformados sobre la vida útil de los productos-, el Parlamento Europeo pide en su mencionada resolución "Hacia un mercado único más sostenible para las empresas y los consumidores" (2020) que, cuando se prepare la revisión de la Directiva 2019/771, se evalúe la forma de ajustar más la duración de las garantías jurídicas a la vida útil estimada de una categoría de productos. No obstante, cabría traer a colación las observaciones antes referidas de Sánchez García (2017), y que podrían resultar igualmente aplicables en el sentido de que una ampliación de los plazos que tenga en cuenta la vida útil de los productos debe hacerse con mucha cautela, fijando unos plazos de vida útil "deseables" y "posibles", y no permitiendo que la vida útil la determinen los comerciantes a través de la obsolescencia programada de los productos. 
No cabe duda de que la extensión de los plazos — se haga de forma general o individualizada según el bien- podría contribuir a una mayor durabilidad de los productos y, consecuentemente, a erradicar la obsolescencia programada. Soto Pineda (2015) lo explica así:

[...] la relación entre la obsolescencia programada y la garantía -fuente de un régimen común adherido a la protección del consumidor-, se fundamenta, principalmente, en el protagonismo que las empresas que ponen en práctica la obsolescencia le dan al periodo de garantía (sea cual sea su determinación en un emplazamiento particular), erigiéndolo como uno de los elementos fundamentales que permiten planificar el tiempo y las circunstancias en las cuales los productos que fabrican devendrán obsoletos. (p. 8) ${ }^{1}$

Resulta interesante, en este punto, la propuesta del autor francés Fabre-Magnan (2017, pp. 384-385), al considerar que la ley debería centrarse más en la calidad de los bienes y servicios ofertados, y que los bienes con obsolescencia programada, o con componentes que no pueden ser reemplazados, podrían no ser puestos en circulación al imponer ciertas normas. Su propuesta no se circunscribiría al derecho de consumo, sino al derecho general de contratos, que podría garantizar un derecho a un producto duradero ("right to a durable product"), a través del sistema de garantías legales y no simplemente contractuales; y de un derecho a un contrato sostenible ("contrat durable"), cuyo objeto y modalidad de cumplimiento permitiría la armonización de sus aspectos económicos, sociales y medioambientales.

\subsection{Deberes para Productos físicos (II): La Reparabilidad}

Otro concepto igualmente importante en materia de sostenibilidad es el de "reparabilidad", sobre todo en relación con la obsolescencia indirecta -imposibilidad de reparación- Cuando un producto deviene defectuoso y la garantía legal ha terminado, el consumidor

1 Véase también Vanina Bianchi, 2018, pp. 299-301. 
aún tiene la posibilidad de reparar el bien por sí mismo o mandarlo a reparar; sin embargo, en la práctica es habitual que aparezcan numerosos obstáculos, como la falta de disponibilidad de piezas de repuesto o el precio desproporcionado de estas (Terryn, 2019, p. 139). En efecto, se ha puesto de manifiesto que:

[...] todos los aparatos modernos, productos de la Smart-industria, resultan extremadamente difíciles de reparar. Este problema técnico, transformado en cuestión social, está dando pie a que se hable de un nuevo derecho, el derecho a la reparación. En Estados Unidos este derecho a la reparación ya se ha colado en la agenda legislativa de algunos Estados [...] El abuso de los fabricantes es creciente: no solo quieren que los clientes tengan que pasar por los intermediarios de reparación autorizados por ellos, sino que además quieren que los productos que ellos venden formen parte de una cadena que genere ventas adicionales. [...] para defender sus tesis anti-reparación los fabricantes afirman que restringiendo la capacidad de reparación no solo se salvaguardan sus derechos de propiedad intelectual, sino que además se protege a los compradores. [...] Pero esto tiene desventajas: los reparadores autorizados son más caros y a veces tampoco saben resolver el problema. (Tráfico y seguridad vial, 2017)

No solo preocupa este abuso por parte de determinadas compañías, sino también ciertas prácticas de información engañosa, por las que se hace creer que se perderá la garantía legal si se lleva a cabo una reparación no autorizada o uno intenta desmontar un dispositivo por sí mismo (Svensson et ál., 2018).

Para promover la durabilidad de los productos, es necesaria una nueva cultura en Europa sobre reparación. Por ello resulta ciertamente reconfortante que el nuevo Plan de Acción para la Economía Circular de 2020 prevea que se trabajará para establecer un nuevo "derecho a la reparación” y que se estudiarán "[...] nuevos derechos sustantivos de carácter horizontal para los consumidores, como la disponibilidad de piezas de recambio, el acceso a servicios de reparación y, en el caso de las TIC y la electrónica, los servicios de actualización". Tal compromiso parece surgir de los reclamos contenidos en la resolución del Parlamento Europeo: “Una vida útil más larga para los productos: ventajas para los consumidores y las empresas" (2017), y que se reitera en la posterior resolución "Hacia un mercado único más sostenible para las empresas y los consumidores (2020)", al afir- 
mar que "El consumo sostenible va de la mano de una producción sostenible", por lo que "[...] debe animarse a los agentes económicos a que consideren la durabilidad de los productos y servicios desde la fase de diseño". Además, en un apartado del mismo documento titulado "Estrategia de reparación", se pide que cierta información relativa a la reparación esté disponible de manera clara y fácilmente comprensible en el momento de la compra, incluyendo un resumen de los fallos más frecuentes y de cómo repararlos.

En definitiva, se urge a la Comisión para que establezca un "derecho de reparación" de los consumidores, con el fin de que las reparaciones sean sistemáticas, rentables y atractivas, llevando a cabo las siguientes medidas: 1) proporcionar a los agentes del sector de la reparación, incluidos los talleres de reparación independientes, y a los consumidores acceso gratuito a la información necesaria sobre la reparación y el mantenimiento; 2) fomentar un proceso de estandarización de las piezas de recambio que favorezca la interoperabilidad y la innovación; 3) establecer un período mínimo obligatorio para el suministro de piezas de recambio que refleje la vida útil estimada del producto; 4) velar por que el precio de una pieza de recambio sea razonable; 5) alentar la reparación en vez de la sustitución, mediante la extensión de las garantías o la reinicialización de los períodos de garantía para los consumidores que se decanten por esta opción; 6) evaluar cómo podrían facilitarse las reparaciones, a través del establecimiento, a escala de la Unión, de una garantía legal para las piezas sustituidas en un taller de reparación profesional; y 7) animar a los Estados miembros a que creen incentivos, como la "bonificación del artesano" que promueven las reparaciones, en particular después del vencimiento de la garantía legal.

Si de verdad se pretende una transición hacia la sostenibilidad y la economía circular, es imprescindible luchar contra la obsolescencia indirecta a través del reconocimiento de un efectivo derecho a la reparación, entendido como el derecho del consumidor a elegir quién, qué, dónde, porqué, cuándo, cómo y por cuánto su producto va a ser reparado (The Repair Association, 2018²).

2 Apartado "How does this impact consumer choice?" de la sección FAQ (Frequently Asked Questions). Disponible en: www.repair.org/stand-up (consultado el 10 de diciembre de 2020). 


\subsection{Deberes para contenidos o SERVICIOS DigitAles: las ACTUALIZACIONES Y LA ADAPTABILIDAD}

La imposición del deber de actualizar los contenidos o servicios digitales se constituye como un remedio frente a la obsolescencia informática. En este sentido cabe señalar que el Parlamento Europeo ya pedía en su Resolución de 4 de julio de 2017, titulada "Una vida útil más larga para los productos: ventajas para los consumidores y las empresas", que se protegiese a los consumidores frente a la obsolescencia de los programas informáticos a través de actualizaciones obligatorias. Esta cuestión ya ha sido abordada en las recientes Directivas 2019/770, relativa a determinados aspectos de los contratos de suministro de contenidos y servicios digitales, y 2019/771, relativa a determinados aspectos de los contratos de compraventa de bienes — en lo que se refiere, en este último caso, a bienes con elementos digitales-. No obstante, el Parlamento Europeo ha vuelto a incidir en esta cuestión en su posterior Resolución de 25 de noviembre de 2020, titulada "Hacia un mercado único más sostenible para las empresas y los consumidores, al afirmar":

[...] la duración de vida útil de los programas informáticos es crucial para la de los aparatos electrónicos; [y] que, toda vez que los programas informáticos quedan obsoletos cada vez con mayor rapidez, es necesario que los aparatos electrónicos sean adaptables para que sigan siendo competitivos.

En nuestra opinión, no debe desestimarse la importancia de la obsolescencia informática, máxime en el momento que atravesamos, y que se caracteriza por una evidente digitalización. Es necesario, pues, que sí se reconozca ese "derecho a productos electrónicos adaptables" para evitar que dichos productos queden rápidamente desfasados e inservibles, en atención a la rapidez con la que evoluciona el sector tecnológico.

\section{VALORACIONES finAlES}

Existen muchas formas de obsolescencia, aunque la más conocida es la programada, sobre todo desde que se comenzó a visibilizar a través de distintas iniciativas — por ejemplo, el documental 
"Comprar, tirar, comprar", del año 2011—; sin embargo, y aunque la percepción sobre ella es manifiesta y genera rechazo, lo cierto es que crea en los consumidores cierto nivel de vacilación y resignación, pues interpretan que es una realidad empresarial indubitada —e incluso hasta necesaria en términos económicos- ante la cual son pocas las acciones que se pueden ejercer (Soto Pineda, 2015, p. 2; Sánchez García, 2017, p. 183-184). Esto, por fortuna, no es cierto. Existe una alternativa posible: la producción y el consumo sostenibles, construidos alrededor de una nueva figura de consumidor responsable y de un sistema de producción circular, en clara contraposición a la economía lineal: extraer, producir, usar y tirar (Kryla-Cudna, 2020, p. 1208).

La construcción de ese modelo de economía sostenible necesita medidas en un amplio abanico de sectores. El problema más acuciante en el momento presente es la inercia; por ello, es necesario repensar la economía, replantearse la sociedad en la que queremos vivir y abrir un debate teórico sobre cuál debe ser el rol que asume el derecho (Micklitz, 2019, p. 345). Derecho que, como ciencia social comportamental que es, puede contribuir a las transformaciones deseadas (Ribeiro Freyesleben y Maas Dos Anjos, 2018, p. 826). Resulta obvio, entonces, que, para alentar la transición hacia una economía circular, los gobiernos necesitan crear las condiciones que estimulen y aceleren las decisiones en este sentido (De Boeck, 2019, pp. 186-187); medidas que se han de tomar en un amplio abanico de sectores (Keirsbilck y Rousseau, 2019, p. 94), incluido, por supuesto, el derecho privado, que debe desempeñar un papel esencial en la transformación.

Para luchar contra la obsolescencia en todas sus formas — programada, funcional, psicológica, informática o indirecta- es necesario proporcionar una mejor información a los consumidores, sobre todo en lo relativo a la durabilidad/ reparabilidad de los productos. Ya en el año 2012, y con motivo del Día Mundial de los Derechos del Consumidor - que cada año se celebra el 15 de marzo y que se había dedicado, en aquella ocasión, al consumo sostenible-, el presidente del Comité Económico y Social Europeo afirmaba en su discurso de apertura que el consumo sostenible requiere que los consumidores desempeñen un papel activo y esencial, y que por ese motivo, el trabajo de los Estados miembros es ayudarles: "Let us give them tools to make smart choices, to be better informed and to make well-reasoned decisions! Let us make sustainable products a real choice for consumers" (Hobson, 2013, pp. 1085-1086). 


\section{ANÁLISIS \\ JURÍDICO-POLÍTICO}

Además de informar mejor a los consumidores, los legisladores también deben fomentar la durabilidad/reparabilidad de los productos extendiendo los plazos de garantía y tomando medidas efectivas para garantizar el derecho a la reparación de los productos o la obligación de que los comerciantes actualicen los bienes con elementos digitales o los contenidos y servicios digitales. Estas propuestas no son en absoluto excesivas, sino acuciantes; recordemos que, según el último Plan de la Unión Europea para la Economía Circular de 2020, en 2050 el consumo mundial será el equivalente al de tres planetas, pero "solo tenemos una Tierra". Conservémosla.

\section{REFERENCIAS}

Bauman, Z. (2006). Vida líquida. Paidós.

Comisión Europea. (2012). Attitudes of Europeans towards building the single market for green products. Oficina de publicaciones

Comisión Europea. (2018). Behavioural Study on consumers' Engagement in the Circular Economy, Reporte final

Comisión Europea, Dirección General de Justicia y Consumidores. (2017). Study on the costs and benefits of extending certain rights under the Consumer Sales and Guarantees Directive 1999/94/EC. Oficina de publicaciones. https: / / data.europa.eu/doi/10.2838/590766

Comité Económico y Social Europeo. (2013, 14 de febrero). Dictamen 2014/C 67/05 (Thierry Libaert, P. y Jean-Pierre Haber, C. P.). Por un consumo más sostenible: la duración de la vida de los productos industriales y la información al consumidor para recuperar la confianza: la duración de la vida de los productos industriales y la información al consumidor para recuperar la confianza. Diario Oficial de la Unión Europea. https: / / bit.ly/2Tk5oDX

Cooper, T. (2004). Inadequate life? Evidence of consumer attitudes to product obsolescence. Journal of Consumer Policy, 27(4), 421-449. https: / / doi.org/10.1007/ s10603-004-2284-6

De Boeck, A. (2019). The 'Circular Economy' - National Legal Initiatives and Challenges Converning Movables - Belgium. En Consumer Protection in a Circular Economy. Cambridge. pp. 185-199.

Fabre-Magnan, M. (2017). What is a Modern Law of Contracts? Elements for a New Manifesto for Social Justice in European Contract Law. European Review of Contract Law, 13(4), 376-388. https:/ / doi.org/10.1515/ ercl-2017-0020

García Goldar, M. (2021). Propuestas para garantizar modalidades de consumo y producción sostenibles (ODS 12). Revista de Fomento Social, 299, 91-114.

Hobson, K. (2013). Weak or strong sustainable consumption? Efficiency, degrowth, and the 10 Year Framework of Programmes. Environment and Planning C: Government and Policy, 31(6), 1082-1098. https:/ / doi.org/10.1068/c12279 
Mónica García Goldar - Tipos de obsolescencia y formas de combatirla desde el derecho privado

Keirsbilck, B. y Rousseau, S. (2019). The Marketing Stage: Fostering Sustainble Consumption Choices in a "Circular" and "Functional" Economy. En Consumer Protection in a Circular Economy. Intersentia. pp. 93-126.

Keirsbilck, B., Terryn, E., Michel, A. y Alogna, I. (2020). Sustainable Consumption and Consumer Protection Legislation: How can sustainable consumption and longer lifetime of products be promoted through consumer protection legislation? Directorate-General for Internal Policies.

Kryla-Cudna, K. (2020). Sales Contracts and the Circular Economy. European Review of Private Law, 28(6), 1207-1230.

Loos, M. B. M. (2017). Not good but certainly content: The proposals for European Harmonisation of Online and Distance Selling of Goods and the Supply of Digital Content. En Digital content $\mathcal{E}$ distance sales: new developments at EU level. Intersentia. pp. 3-53.

Mak, V. y Terryn, E. (2020). Circular Economy and Consumer Protection: The Consumer as a Citizen and the Limits of Empowerment Through Consumer Law. Journal of Consumer Policy, 43, 227-248. https: / / doi.org/10.1007/ s10603-01909435-y

Mazeika, R. (2019). Consumer Protection in the Circular Economy in Europe - Legal Initiatives and Challenges - The Commission's View. En Consumer Protection in a Circular Economy. Intersentia. pp. 11-18.

Mesacasa, A., Corona, H. M. P. y Mello, N. A. (2010). Ecodesign: um novo paradigma moderno inscrito entre moda e consumo? Design $\mathcal{E}$ Tecnologia, 1(2), 1-7. https: / / doi.org/10.23972/ det2010iss02pp1-7

Micklitz, H-W. (2019). Squaring the Circle? Reconciling Consumer Law and the Circular Economy. En Consumer Protection in a Circular Economy. Intersentia. pp. 323-345.

Nascimento Pereira, M. (2017). Consumo sustentável: a problemática da obsolescência programada e o descarte de produtos. Revista Eletrônica Direito e Sociedade, 5(2), 209-220.

Martínez, A. y Porcelli, A. (2016). Un difícil camino en pos del consumo sustentable: el dilema entre la obsolescencia programada, la tecnología y el ambiente. Lex: Revista de la Facultad de Derecho y Ciencia Política de la Universidad Alas Peruanas, 14(18), 333-378.

Pantzar, M. y Suljada, T. (2020). Delivering a circular economy within the planet's boundaries: An analysis of the new EU Circular Economy Action Plan. Institute for European Environmental Policy (IEEP); Stockholm Environment Institute (SEI). https: / / bit.ly / 2URNMQ8

Pazos, R. (2020). Sustainability, the Circular Economy and Consumer Law in Spain. Journal of European Consumer and Market Law, 9(5), 212-215.

Ribeiro Freyesleben, L. E. y Maas dos Anjos, R. (2018). Circularidad en tiempos obsoletos. En Congreso Nacional del Agua Orihuela: Innovación y Sostenibilidad. Universitat d'Alacant. pp. 819-830.

Richter, J. L. y Dalhammar, C. (2019). Stakeholders, drivers and barriers for local electronics repair: a case study of southern Sweden. En Product Lifetimes and the Environment 2019 Conference Proceedings. 


\section{ANÁLISIS \\ JURÍDICO-POLÍTICO}

Sánchez García, M. (2017). La obsolescencia programada en las prácticas comerciales y su relación con el régimen de vicios ocultos y garantías de consumo. En Aproximaciones jurídicas a la obsolescencia programada. Universidad Externado de Colombia. pp. 169-198.

Santamaría Arinas, R. J. (2019). Economía circular: líneas maestras de un concepto jurídico en construcción. Revista Catalana de Dret Ambiental, 10(1), 1-37. https: / / doi.org/10.17345/rcda2567

Schally, H. M. (2019). The Circular Economy Action Plan: An Agenda for Change. En Consumer Protection in a Circular Economy. Intersentia. pp. 3-9.

Soto Pineda, J. A. (2015). Reflexiones acerca de las posibles incompatibilidades de la obsolescencia programada con el sistema de defensa de los consumidores. Actualidad Civil, 6, pp. 40-55.

Svensson, S., Richter, J. L., Maitre-Ekern, E., Pihlajarinne, T., Maigret, A. y Dalhammar, C. (2018). The Emerging "Right to Repair" legislation in the EU and the U.S. Ponencia presentada en Going Green CARE INNOVATION 2018, Vienna, Austria.

Terryn, E. y Van Gool, E. (2020, 18 de noviembre). The role of European consumer regulation in shaping the environmental impact of e-commerce (working paper). Journal of European Consumer and Market Law. http:/ / dx.doi. org/10.2139/ssrn.3732911

Terryn, E. (2019). A Right to Repair? Towards Sustainable Remedies in Consumer Law. En Consumer Protection in a Circular Economy. Intersentia. pp. 127-147.

Van Acker, K. (2019). Technology for Circular Economy. A New Paradigm for the Way We Use Resources. Wn Consumer Protection in a Circular Economy. Intersentia. pp. 21-32.

Vanina Bianchi, L. (2018). La influencia del principio del consumo sustentable en el combate de la obsolescencia programada, la garantía de los productos durables y el derecho a la información de los consumidores en Argentina. Revista de Derecho Privado, 34(enero-junio), 277-310. https: / / doi.org/10.18601/01234366.n34.09

Vidalenc, E. y Meunier, L. (2014). Obsolescence des produits: l'impact écologique. Revue Futuribles, 402, 5-21. 